\title{
Primitive neuroectodermal tumor mimicking left atrial myxoma
}

\author{
Kinnaresh Baria ${ }^{\mathrm{a}, *}$, Amber Malhotra $^{\mathrm{b}}$, Kartik Patel ${ }^{\mathrm{a}}$, Pratik Shah ${ }^{\mathrm{b}}$, Sumbul Siddiqui ${ }^{\mathrm{a}}$ \\ a Department of Cardiovascular and Thoracic Surgery, U. N. Mehta Institute of Cardiology and Research Center (Affiliated to BJ Medical College, Ahmedabad), India \\ b Department of Research, U. N. Mehta Institute of Cardiology and Research Center (Affiliated to BJ Medical College, Ahmedabad), India
}

\section{Introduction}

Primary cardiac tumours are rare; the incidence ranges from $0.001 \%$ to $0.28 \%{ }^{1}$ Malignant tumours account for $25 \%$ of primary cardiac tumours. ${ }^{1}$ Primary primitive neuroectodermal tumour (PNET) is derived from carcinogenic alteration of pluripotent neural crest cells and rarely presents as an organ-based neoplasm. ${ }^{2}$ We present a rare case of primary PNET of the heart.

\section{Case history}

An 18-year-old boy presented with a 3 months' history of progressively worsening breathlessness and peripheral oedema. There was no significant family history. Echocardiography demonstrated a $58 \mathrm{~mm} \times 49 \mathrm{~mm}$ mass, probably left atrial myxoma with no pericardial effusion and good left ventricular function. Myxoma removal was planned two days after echocardiography evaluation, intraoperatively there was firm vascular mass present in left atrium with involvement of interatrial septum and extending into right pulmonary veins, the mass was encasing aorta and pulmonary artery on lateral and posterior surface with involvement of pericardium anteriorly, so procedure was abandoned after incisional biopsy which was sent for immunohistochemistry and histopathological examination (HPE) (Fig. 1). Post-operative high resolution contrast computerized tomography imaging (Fig. 2) revealed evidence of large, lobulated ill-defined multicompartment, heterogeneously enhancing mediastinal nodal mass. The mass had non-enhancing necrotic areas within, seen contiguously involving left lower cervical, aorto-pulmonary window, pre-vascular, pre-subcarinal and bilateral hilar regions. This solid seemingly encapsulated mass was adherent to left atrium and appeared to arise from the posterior free wall of the left atrium. A vascular, nonhomogeneous mass was seen to be densely adherent to the posterior wall of the left atrium and the coronary sinus. HPE report was suggestive of tumour infiltrating the myocardium which was composed of solid sheets of atypical cells with vesicular nuclei, variably visible small nucleoli, and eosinophilic cytoplasm. Mitoses were seen and there was focal tumour necrosis. An associated brisk lymphocytic infiltrate was present in places. No evidence of gland formation, mucin production, or keratinization was

\footnotetext{
* Corresponding author at: Department of Cardio Vascular and Thoracic Surgery, U. N. Mehta Institute of Cardiology and Research Center, Civil Hospital Campus, Asarwa, Ahmedabad 380016, Gujarat, India.

E-mail address: drkinnaresh@gmail.com (K. Baria).

Peer review under responsibility of The Society of Cardiovascular Academy.
}

apparent. Immunostaining revealed the tumour to be positive for CD 99 (Cluster of Differentiation 99) and vimentin. The tumour was negative for lymphoid markers (leukocyte common antigen, CD 20), endothelial markers (CD 31, CD 34). In view of the immunoprofile, including positivity for CD 99, the diagnosis of PNET was made. The patient was transferred to oncology unit on the sixth postoperative day for oncologic review. Chemotherapy with cyclophosphamide and radiotherapy with 5000 rads per cycle, a total of five cycles were given. A post chemotherapy computerized tomography confirmed tumour shrinkage and no evidence of tumour involvement elsewhere.

\section{Discussion}

We present a case of primary PNET of the heart being diagnosed as LA myxoma on echocardiography, received chemo-radiotherapy for the same but ultimately, he succumbed after six months of diagnosis. Since no evidence of tumour was found elsewhere, we propose that this represents a primary PNET of the left atrial free wall. Primary cardiac tumours are rare; the incidence ranges from $0.001 \%$ to $0.28 \% .{ }^{1}$ Malignant tumours account for $25 \%$ of primary cardiac tumours. ${ }^{1}$ The most frequent primary malignant cardiac tumours are angiosarcomas. Primitive neuroectodermal tumour derives from carcinogenic alteration of

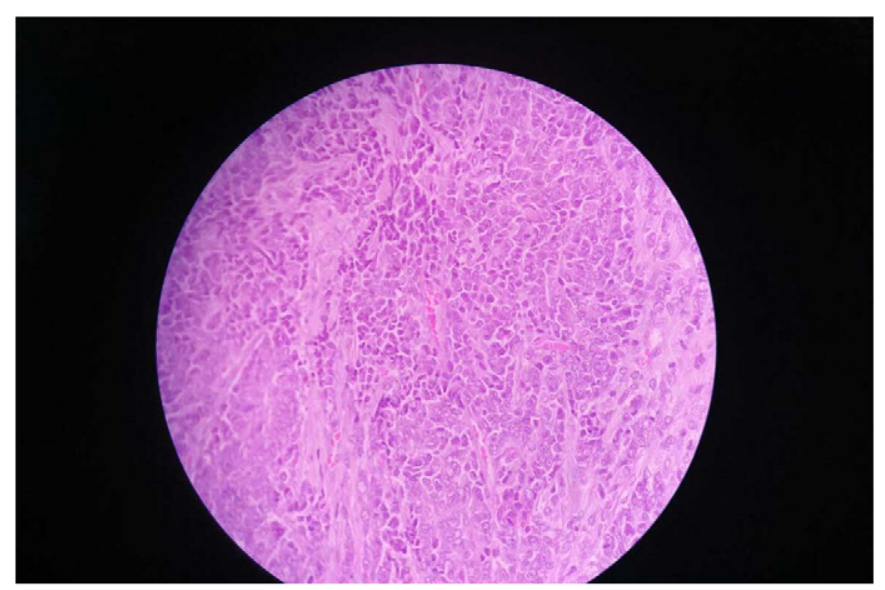

Fig. 1. Histopathological examination image of mass showing solid sheet of atypical cells with vesicular nuclei. 


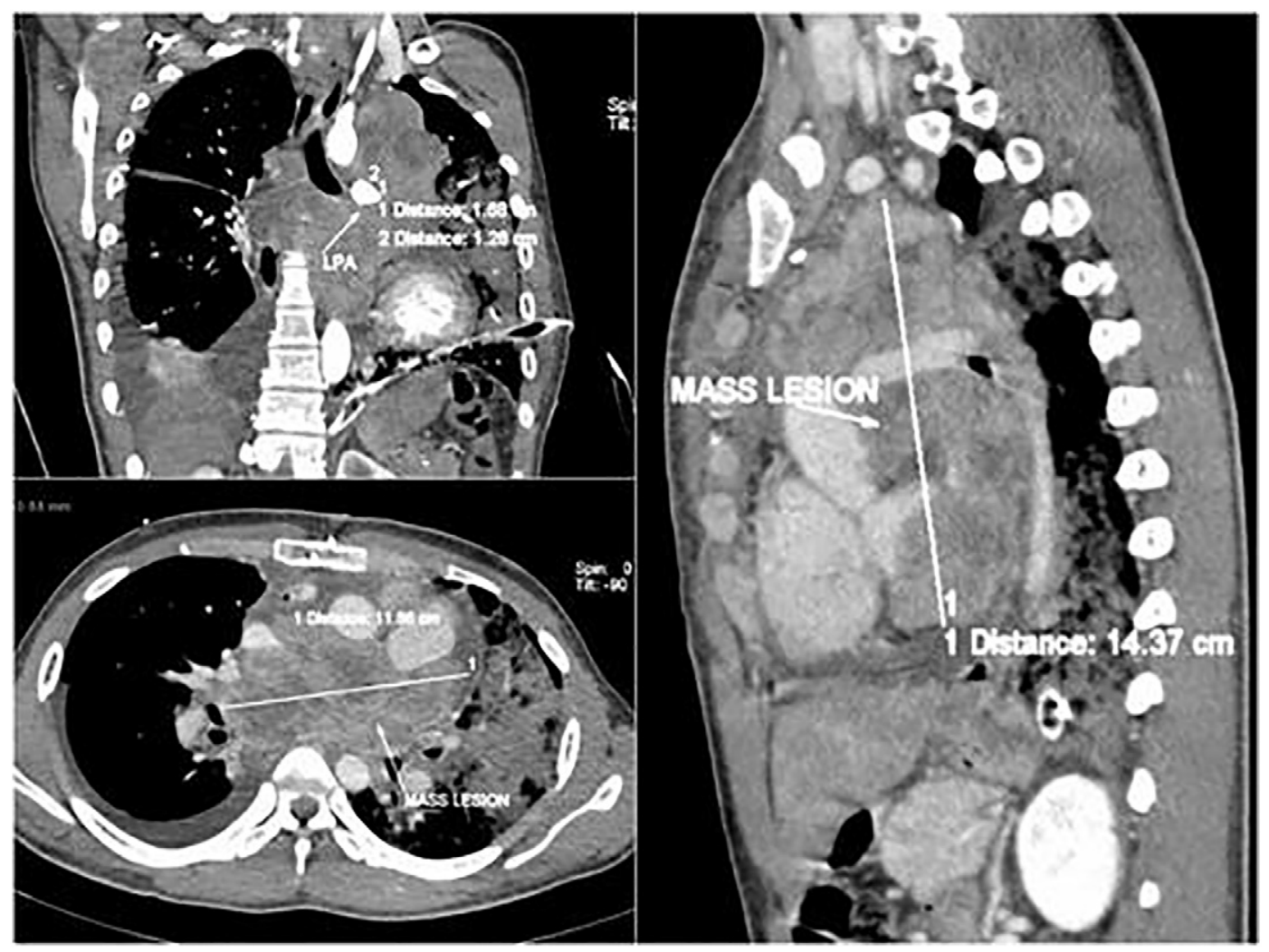

Fig. 2. Computerised tomography scan of thorax suggestive of mass lesion encasing pulmonary artery and superior vena cava.

pluripotent neural crest cells through a balanced reciprocal translocation $\mathrm{t}(11 ; 22)(\mathrm{q} 24 ; \mathrm{q} 12) .^{2}$ It rarely presents as an organ-based neoplasm and is typically seen in the soft tissues of the chest wall and paraspinal region. ${ }^{2}$ There is no clear guidance on the treatment of PNET of the heart. Cardiac neural crest cells participate in endocardial cushion and the septation of the cardiac outflow tract into aorta and pulmonary artery. We acknowledge the possibility that this could be a cardiac metastasis behaving like a primary lesion because primary tumours can undergo spontaneous regression after treatment of metastases. ${ }^{3}$ But as there was no evidence of gland formation, mucin production, or keratinization was on HPE, possibility of adenocarcinoma and squamous cell carcinoma can be ruled out. In a 10-year-period review of 18,751 post-mortem examinations, Bussani and associates ${ }^{1}$ discovered 7289 cases (39\%) of previously undiagnosed malignant neoplasm. Six hundred and twenty-two of these showed evidence of cardiac metastasis (9.1\% of malignant tumours). However, the heart was found to be the only target of metastasis in 10 cases $(10 / 7289$; $0.14 \%$ ). The highest rates of cardiac metastases are seen from pleural mesothelioma (48.4\%), melanoma (27.8\%), lung adenocarcinoma (21\%), undifferentiated carcinoma (19.5\%), lung squamous cell carcinoma (18.2\%), and breast carcinoma (15.5\%). ${ }^{1}$ PNET of heart carries a poor prognosis. Myxomas are typically pedunculated, with a stalk that is attached to the interatrial septum. For echocardiographically atypical tumours a differential diagnosis should be considered by doing a detailed echocardiography before labelling it as myxoma.

\section{Sources of funding for the work}

Nil.

\section{Conflicts of interest}

Nil.

\section{References}

1. Bussani R, De-Giorgio F, Abbate A, Silvestri F. Cardiac metastases. J Clin Pathol 2007;60: 27-34.

2. Besirli K, Arslan C, Tuzun H, Oz B. The primitive neuroectodermal tumour of the heart Eur J Cardiothorac Surg 2000;18:619-621.

3. Fairlamb DJ. Spontaneous regression of metastases of renal cancer: a report of two cases including the first recorded regression following irradiation of a dominant metastasis and review of the world literature. Cancer 1981;47:2102-2106. 